\title{
A ROBUST OVERRIDE SCHEME ENFORCING STRICT OUTPUT CONSTRAINTS FOR A CLASS OF STRICTLY PROPER SYSTEMS
}

\author{
Guido Herrmann, Matthew C. Turner and Ian Postlethwaite
}

\author{
Control and Instrumentation Group, University of Leicester, University \\ Road, Leicester, LE1 7RH, UK, Tel.: ++44-116-252 2567, \\ e-mail: gh17@le.ac.uk, mct6@le.ac.uk, ixp@le.ac.uk
}

\begin{abstract}
This paper presents an override controller which ensures that constrained output variables retain certain prescribed strict bounds. The class of nominal closed loop systems considered for the constrained output regulation problem is strictly proper and minimum phase, assuming for each output measurement constraint one available actuator and the first Markov parameter to be full rank. This necessitates the open-loop plant to have the same input-toconstrained-output characteristics. The advantage of the considered class of nominal systems is that an output constraint translates directly into a state constraint for which it is possible to use a particular non-smooth Lyapunov function. The non-smooth Lyapunov function is defined by the level of the output constraint creating an invariant set for which the strict output constraints are satisfied. The override strategy is designed to retain a minimal effect on the nominal control loop in case no output constraint is violated.

Copyright 2005 IFAC
\end{abstract}

Keywords: Output constraint, override control, non-smooth Lyapunov function, high-gain component

\section{INTRODUCTION}

Engineers usually design controllers using conventional design techniques and these controllers operate well in a reasonable operating region of the closed loop system. However, in practical systems, strict constraints of a plant have to be enforced, such as mechanical stops in mechatronic systems which define clear limits either to output signals or the actuator input of a plant. Among those two issues, the problem of output signal constraints has been of lesser interest, although a significant amount of publications exists: Conceptual results have been presented by Saberi et al. (2002) giving conditions on the existence of controllers for constrained systems. Other work aimed at controller design can be grouped into four areas: control using the 'maximum output admissible set' (Gilbert and Tan, 1991), model predictive control (Bemporad et al., 2003), invariance control (Mareczek et al., 2000; Mareczek et al., 2001; Mareczek et al., 2002; Wolff and Buss, 2004) and override control (Glattfelder and Schaufelberger, 1988; Turner and Postlethwaite, 2002; Glattfelder and Schaufelberger, 2003). The method for control using the 'maximum output admissible set' can be computationally very expensive as it is the aim to compute at first the maximum reachable set assuming certain plant input and output constraints. Similarly, it can be computationally costly to use model predictive ideas as it is necessary to calculate a time varying controller valid for a certain finite horizon in advance to the controller operation. For the work by Wolff and Buss (2004), the method of invariant control is strongly related to the idea of the 'maximum output admissible set' as control signal limits are assumed and an 'admissible set' is retained invariant by employing a suitably chosen control law. Wolff and Buss's (2004) scheme can be also regarded as an override controller, since an add-on controller is devised in addition to a nominal control scheme to retain amplitude boundedness. Wolff and Buss (2004) can assure for their scheme strict attainment of the amplitude limits using a discontinuous control law. In this sense, the paper by Wolff and Buss (2004) considers a special class of non-linear single-input full-information feedback systems without strict consideration of disturbances. This severely contrasts the approaches by Glattfelder and Schaufelberger (1988), Glattfelder and Schaufelberger (2003) and in particular by Turner and Postlethwaite (2002). There it is easily possible to consider large scale multi-input-multi-output plants for output feedback control, disturbances and more than one output measurement constraint, while only 'soft output limits' are enforced. Thus, it is the approach of this paper to develop an override scheme which can strictly retain output measurement limits for multi-input multioutput systems. The scheme is robust to bounded disturbances assuming that the bounds are known.

This paper employs two main principles: a nonsmooth Lyapunov function, termed max-Lyapunov function (Herrmann et al., 1999; Herrmann, 2000), (see (Blanchini, 1995) for similar ideas) and a high gain control approach. The non-smooth max-Lyapunov function with a partially polyhedral level set has the advantage that the output constraints for strictly proper 
plants can be directly expressed in terms of a specific stability condition for the max-Lyapunov function: Since it is possible to convert the output constraints for strictly proper plants directly into state constraints, the shape of a level set of the max-Lyapunov function can be defined by the level of the output constraints. Hence, once the max-Lyapunov function has a value larger than the output constraint limit, a high gain control law is activated which assures for bounded output demand and bounded disturbance the strict limit on the output measurements. As some ideas of this high gain controller relate strongly to a sliding mode control output feedback approach by Edwards and Spurgeon (1998, Chapter 5), the same class of strictly proper plants but a reasonably arbitrary class of linear controllers is considered.

\section{NOTATION}

To describe controller constraints, a multi-variable saturation Sat : $\mathbb{R}^{n_{s}} \mapsto \mathbb{R}^{n_{s}}$ and a deadzone function $\mathrm{DZ}: \mathbb{R}^{n_{s}} \mapsto \mathbb{R}^{n_{s}}$ are essential:

$$
\begin{aligned}
& \operatorname{Sat}(\boldsymbol{s})=\left[\operatorname{sat}\left(s_{1}\right) \operatorname{sat}\left(s_{2}\right) \ldots \operatorname{sat}\left(s_{n_{s}}\right)\right]^{T}, \\
& \mathrm{DZ}(\boldsymbol{s})=\left[\begin{array}{llll}
\mathrm{Dz}\left(s_{1}\right) & \mathrm{Dz}\left(s_{2}\right) \ldots \mathrm{Dz}\left(s_{n_{s}}\right)
\end{array}\right]^{T} \\
& =I-\operatorname{Sat}(s) \\
& \text { where } \boldsymbol{s}=\left[\begin{array}{lllll}
s_{1} & s_{2} & s_{3} & \cdots & s_{n_{s}}
\end{array}\right]^{T} \text { and } \\
& \operatorname{sat}\left(s_{i}\right)=\max \left(\min \left(s_{i}, 1\right),-1\right) \text {. }
\end{aligned}
$$

Furthermore, the following non-linear discontinuous switching function $U: \mathbb{R}^{n_{s}} \mapsto \mathbb{R}$ will be of interest:

$$
U(\boldsymbol{s})=\left\{\begin{array}{l}
1 \text { for } \Sigma_{i=1}^{n_{s}}\left(\left|\mathrm{Dz}\left(s_{i}\right)\right|\right)>0 \\
0 \text { for } \Sigma_{i=1}^{n_{s}}\left(\left|\mathrm{Dz}\left(s_{i}\right)\right|\right)=0
\end{array} .\right.
$$

In addition, the function $\operatorname{sign}(\cdot)$ for a real scalar $s_{i}$ is defined as:

$$
\operatorname{sign}\left(s_{i}\right)=\left\{\begin{array}{c}
1 \text { for } s_{i}>0 \\
0 \text { for } s_{i}=0 \\
-1 \text { for } s_{i}<0
\end{array}\right.
$$

\section{THE UNCONSTRAINED CLOSED LOOP CONTROL SYSTEM}

Assume the closed loop system, combining controller and plant, is defined as:

$$
\begin{aligned}
\dot{\boldsymbol{x}} & =A \boldsymbol{x}+B_{w} \boldsymbol{w}+B_{u} \boldsymbol{u} \\
\boldsymbol{z} & =C_{z} \boldsymbol{x} \\
\boldsymbol{y} & =C_{y} \boldsymbol{x}
\end{aligned}
$$

where $\boldsymbol{x} \in \mathbb{R}^{n}$ is the closed loop system state and $\boldsymbol{u} \in \mathbb{R}^{n_{u}}$ is the override control input, $\boldsymbol{w} \in \mathbb{R}^{n_{w}}$ is the exogenous input, $\boldsymbol{y} \in \mathbb{R}^{n_{y}}$ is the measurement output and $z \in \mathbb{R}^{n_{z}}, n_{z}=n_{u} \leq n$, is the performance output, which is amplitude constrained and for which the first Markov parameter is non-singular:

$$
\operatorname{rank}\left(C_{z} B_{u}\right)=n_{z} \text {. }
$$

The input-output behavior $\boldsymbol{u} \mapsto \boldsymbol{z}$ is minimum-phase. ${ }^{\dagger}$ Furthermore, the performance output $z$ is measurable, hence, the range of $C_{z}^{T}$ is a subset of the range of $C_{y}^{T}$ so that:

$$
\operatorname{rank}\left[\begin{array}{c}
C_{z} \\
C_{y}
\end{array}\right]=\operatorname{rank}\left(C_{y}\right)
$$

\footnotetext{
$\dagger$ Due to the existence of an exogenuous disturbance, it is easily verified from Saberi et al. (2002) that the minimum phase constraint is necessary for the presented output constrained system.

Assuming that $z$ is a direct plant output measurement, it is easily verified that the plant has to have the same input-output characteristics for the map $\boldsymbol{u} \mapsto z$
}

It is assumed that the closed loop system has been scaled so that the amplitude limit is 1 for all outputs $z_{i}, \boldsymbol{z}=\left[\begin{array}{llll}z_{1} & z_{2} & \cdots & z_{n_{z}}\end{array}\right]^{T}$. This limit may be regarded as a strict limit, where the override controller has to be activated to constrain the output signal of the closed loop system of plant and controller. It is assumed that the closed loop system matrix $A$ is Hurwitz. Furthermore, strict limits for the exogenous signals $\boldsymbol{w}=\left[\begin{array}{llll}w_{1} & w_{2} & \cdots & w_{n_{w}}\end{array}\right]^{T}$ are known:

$$
\left|w_{i}\right| \leq L_{i}, i=1, \cdots, n_{w}
$$

There exists a positive definite matrix $P_{s} \in \mathbb{R}^{n \times n}$ defining a Lyapunov function $V_{s}(\boldsymbol{x})=\boldsymbol{x}^{T} P_{s} \boldsymbol{x}$, so that

$$
P_{s} A+A^{T} P_{s}<0 \text {. }
$$

From Edwards and Spurgeon (1998, Section 5.3), it follows that the linear system can be represented in the following structure (employing a suitable linear transformation):

$$
\begin{aligned}
{\left[\begin{array}{c}
\dot{\boldsymbol{x}}_{1} \\
\dot{\boldsymbol{x}}_{2}
\end{array}\right] } & =\left[\begin{array}{l}
A_{11} \mid A_{12} \\
\hline A_{21} \mid A_{22}
\end{array}\right]\left[\begin{array}{l}
\boldsymbol{x}_{1} \\
\boldsymbol{x}_{2}
\end{array}\right]+\left[\begin{array}{l}
B_{w 1} \\
B_{w 2}
\end{array}\right] \boldsymbol{w}+\left[\frac{B_{u 1}}{0}\right] \boldsymbol{u} \\
\boldsymbol{z} & =\left[I_{n_{z}} 0\right]\left[\begin{array}{l}
\boldsymbol{x}_{1} \\
\boldsymbol{x}_{2}
\end{array}\right], \quad \boldsymbol{x}=\left[\begin{array}{l}
\boldsymbol{x}_{1} \\
\boldsymbol{x}_{2}
\end{array}\right]
\end{aligned}
$$

where $I_{n_{z}} \in \mathbb{R}^{n_{z} \times n_{z}}$ is an identity matrix and $B_{u 1} \in$ $\mathbb{R}^{n_{u} \times n_{u}}$ is full rank. The eigenvalues of the matrix $A_{22}$ represent the stable invariant zeros of the closed loop system (Edwards and Spurgeon, 1998, Lemma 5.1).

\section{AN OVERRIDE CONTROL STRATEGY USING A NON-SMOOTH LYAPUNOV FUNCTION}

The control input $\boldsymbol{u}$ in (1) is to be used to satisfy the amplitude limits of $z \in \mathbb{R}^{n_{z}}$ by introducing an override control law:

$$
\begin{aligned}
\boldsymbol{u}= & M_{1} \boldsymbol{y}+\underbrace{M_{2} \boldsymbol{y} U\left(\frac{\boldsymbol{z}}{\mu}\right)}_{\boldsymbol{u}_{o 1}} \\
& -\underbrace{B_{u 1}^{-1}\left[\begin{array}{ccc}
k_{1} \operatorname{sign}\left(\tilde{d}_{1}\right) & 0 & 0 \\
0 & \vdots & 0 \\
0 & 0 & k_{n_{u}} \operatorname{sign}\left(\tilde{d}_{n_{u}}\right)
\end{array}\right]}_{\boldsymbol{u}_{o 2}}, \tilde{d}_{i}=\operatorname{Dz}\left(z_{i}\right),
\end{aligned}
$$

where $0<\mu<1$ and $M_{i} \in \mathbb{R}^{n_{u} \times n_{y}}, i=1,2$. The override control law introduces two non-linear components, a switched linear term $\boldsymbol{u}_{o 1}$ and a high gain component $\boldsymbol{u}_{o 2}$. The controller term of $\boldsymbol{u}_{o 2}$ is mainly responsible for enforcing the output limits employing a high gain controller, i.e. it is only enabled once the output signals $z$ are at the absolute amplitude limit of 1 . The other components are introduced to retain asymptotic stability. In particular, the linear component $M_{1} y$ is employed for this reason, acting all the time. Thus, the component actually modifies the nominal control system (1). Nevertheless, it has to be assured that the control term $M_{1} \boldsymbol{y}$ affects the control system as little as possible. Hence, the control law has to satisfy an asymptotic stability constraint for vanishing exogenous signal $\boldsymbol{w}=0$ and amplitude boundedness of the output signal $\boldsymbol{z}$ for a suitable initial value $\boldsymbol{x}(0)$ and limited $\boldsymbol{w}$ (4) while retaining the influence of $M_{1} \boldsymbol{y}$ small.

The general idea for ensuring these constraints is to utilize a non-smooth Lyapunov function which assures directly that the constrained output $z$ remains limited to 
the interval $[-1,1]$ for each of the $n_{z}$ elements. This is possible since the squared values of the elements of $z$ determine the value of the non-smooth 'maxLyapunov' function $V_{z}: \mathbb{R}^{n_{z}} \mapsto \mathbb{R}$ :

$$
\begin{aligned}
& V_{z}=\max _{1 \leq i \leq n_{z}}\left(V_{i}\right), \\
& V_{1}=z_{1}^{2}, V_{2}=z_{2}^{2}, \cdots V_{n_{z}}=z_{n_{z}}^{2},
\end{aligned}
$$

so that ensuring $V_{z}<1$ for all disturbances $\boldsymbol{w}$ and suitable initial value $\boldsymbol{x}(0)$ assures the control constraint is satisfied. However, for stability of the override scheme, it will be necessary to consider a max-Lyapunov function $V_{\boldsymbol{x}}: \mathbb{R}^{n} \mapsto \mathbb{R}$ which considers the whole state vector $\boldsymbol{x}$ :

$$
\begin{aligned}
& V_{\boldsymbol{x}}=\max \left(V_{z}, V_{n_{z}+1}\right)=\max _{1 \leq i \leq n_{z}+1}\left(V_{i}\right), \\
& V_{n_{z}+1}=\boldsymbol{x}_{2}^{T} P_{\boldsymbol{x}_{2}} \boldsymbol{x}_{2}
\end{aligned}
$$

for $P_{x_{2}}=P_{x_{2}}^{T} \in \mathbb{R}^{n-n_{z}}, P_{x_{2}}>0$. This max-Lyapunov function is used to prove asymptotic stability in case no exogenous input $\boldsymbol{w}$ has an effect on the closed loop system. Note that $V_{x}$ is positive definite and radially unbounded in $\boldsymbol{x}$, while $V_{z}$ is positive semi-definite in $\boldsymbol{x}$. Both functions will be used as Lyapunov functions, although they are non-smooth.

Asymptotic stability: Without going into the details of non-smooth analysis (Clarke et al., 1998; Herrmann, 2000; Blanchini, 1995; Craven, 1982) and the problem of differential equations with discontinuous right hand sides (Filipov, 1964), the necessary ideas for stability are stated in its most simple way: Since a switching controller will be used, it will be of interest to show for $\boldsymbol{w}=0$ that for almost all $t \geq 0$ :

$$
\frac{d V_{x}}{d t}<0 \text { for } V_{x}>\mu, \mu<1 .
$$

The max-Lyapunov function $V_{x}$ is absolutely continuous and differentiable almost everywhere in $t$. The relationship of (9) can be shown by investigating every single function $V_{i}\left(1 \leq i \leq n_{z}+1\right)$ proving that (Herrmann, 2000, Theorem 4.1):

$$
\frac{d V_{i}}{d t}<0 \text { for } V_{\boldsymbol{x}}>\mu, V_{\boldsymbol{x}}=V_{i} .
$$

This assures in a first step ultimate boundedness for the set $\left\{\boldsymbol{x} \mid V_{\boldsymbol{x}} \leq \mu\right\}$. The override component $M_{1} \boldsymbol{y}+$ $\boldsymbol{u}_{o 1}(5)$ is responsible for this. Asymptotic stability is assured by imposing another stability constraint which is enforced by suitably designing $M_{1} \boldsymbol{y}+\boldsymbol{u}_{o 1}$ (5) for $\boldsymbol{x} \in\left\{\boldsymbol{x} \mid 0<V_{\boldsymbol{x}}(\boldsymbol{x})<1\right\}$ :

$$
\frac{d V_{s}}{d t}<0, V_{s}=\boldsymbol{x}^{T} P_{s} \boldsymbol{x}
$$

for a positive definite matrix $P_{s} \in \mathbb{R}^{n \times n}$. This procedure will ensure that the origin in the disturbance free system is asymptotically approached: The Lyapunov function $V_{s}$ remains asymptotically decreasing in $t$ for any $\boldsymbol{x} \in\left\{\boldsymbol{x} \mid 0<V_{\boldsymbol{x}}(\boldsymbol{x})<1\right\}$. However, $V_{\boldsymbol{x}}$ is shown to be ultimately smaller than or equal to $\mu$ as $V_{x}$ remains a decreasing function of $t$ for $\left\{\boldsymbol{x} \mid V_{\boldsymbol{x}}>\mu\right\}$. Since the sets $\left\{\boldsymbol{x} \mid V_{\boldsymbol{x}}>\mu\right\}$ and $\left\{\boldsymbol{x} \mid 0<V_{\boldsymbol{x}}(\boldsymbol{x})<1\right\}$ are overlapping, asymptotic stability is assured.

Amplitude boundedness: The reduced max-Lyapunov function of (6) is of interest:

$$
V_{z}=\max _{1 \leq i \leq n_{z}}\left(V_{i}\right)
$$

which has to remain decreasing in $t$ for $\boldsymbol{x} \in\left\{\boldsymbol{x} \mid V_{z}>\right.$ $1\}$ :

$$
\frac{d V_{z}}{d t}<0
$$

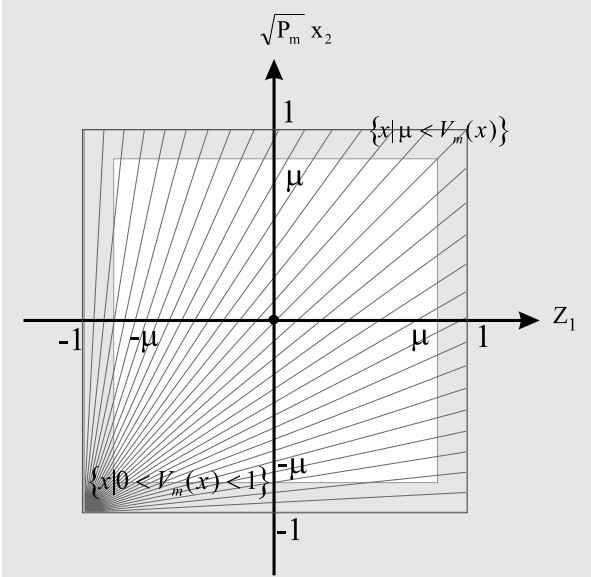

Fig. 1. Set $\left\{\boldsymbol{x} \mid 0<V_{\boldsymbol{x}}(\boldsymbol{x})<1\right\}$ (striped pattern) and set $\left\{\boldsymbol{x} \mid V_{\boldsymbol{x}}>\right.$ $\mu\}$ (grey plain) for $n=2$ and $n_{u}=n_{z}=1$

for limited exogenous input $\boldsymbol{w}(4)$ and small enough unconstrained states $\boldsymbol{x}_{2}$ using $V_{n_{z}+1}(9)$ :

$$
V_{n_{z}+1}=\boldsymbol{x}_{2}^{T} P_{\boldsymbol{x}_{2}} \boldsymbol{x}_{2} \leq L_{m}^{2} .
$$

The high gain component $\boldsymbol{u}_{o 1}$ (5) is used to assure this. Optimization for minimal effect of $M_{1} \boldsymbol{y}$ : An $\mathcal{L}_{2}$-gain relationship is introduced to minimize $M_{1}$ as well as the effect of $\boldsymbol{u}_{o 1}$. At the same time, a sector bound is employed for $\boldsymbol{y} U\left(\frac{z}{\mu}\right)$ from $\boldsymbol{u}_{o 1}$ (5) to assure in particular the stability constraint of (11). For this reason, note that $\boldsymbol{s} \stackrel{\text { def }}{=} \boldsymbol{y} U\left(\frac{z}{\mu}\right)$ satisfies the following inequality:

$$
\boldsymbol{s}^{T} W(\boldsymbol{y}-\boldsymbol{s}) \geq 0
$$

for an arbitrary diagonal positive definite matrix $W \in$ $\mathbb{R}^{n_{m} \times n_{m}}$. Thus, the stability constraint of (11) is extended to:

$$
\begin{aligned}
& \frac{d V_{s}}{d t}+2 \boldsymbol{s} W(\boldsymbol{y}-\boldsymbol{s}) \\
& +\frac{1}{\gamma}\left(M_{1} \boldsymbol{y}+M_{2} \boldsymbol{s}\right)^{T}\left(M_{1} \boldsymbol{y}+M_{2} \boldsymbol{s}\right)-\gamma \boldsymbol{w}^{T} \boldsymbol{w}<0,
\end{aligned}
$$

for any $\left[\boldsymbol{x}^{T} \boldsymbol{s}^{T} \boldsymbol{w}^{T}\right] \neq 0$ using the $\mathcal{S}$-procedure (Boyd et al., 1994). Considering all these stability and output constraints, the main result of the paper can be summarized in the following theorem:

Theorem 1. The override controller of (5) assures that $(i)$ the disturbance free system $(1)-(3)(\boldsymbol{w}=0)$ is globally asymptotically stable.

(ii) the override component $M_{1} \boldsymbol{y}+M_{2} \boldsymbol{y} U\left(\frac{z}{\mu}\right)$ has for the map $\boldsymbol{w} \mapsto M_{1} \boldsymbol{y}+M_{2} \boldsymbol{y} U\left(\frac{z}{\mu}\right)$ an $\mathcal{L}_{2}$-gain of less than $\gamma$ (minimizing the effect of the override compensator for $\boldsymbol{x} \in\left\{\boldsymbol{x} \mid V_{z}(\boldsymbol{x}) \leq 1\right\}$ on the nominal closed loop), provided that the following $n_{z}+2$ matrix inequalities are satisfied

$$
\left[\begin{array}{l|l|l}
P_{s} A+P_{s} B_{u} M_{1} C_{y}+A^{T} P_{s} & P_{s} B_{u} M_{2} \\
+C_{y}^{T} M_{1}^{T} B_{u}^{T} P_{s}+\frac{1}{\gamma} C_{y}^{T} M_{1}^{T} M_{1} C_{y} & P_{s} B_{w} & \begin{array}{l}
+C_{y}^{T} M_{1}^{T} M_{2} \\
\gamma \\
+C_{y}^{T} W
\end{array}
\end{array}<0\right.
$$

$E_{i}\left(A+B_{u}\left(M_{1}+M_{2}\right) C_{y}\right)+\left(A^{T}+C_{y}^{T}\left(M_{1}+M_{2}\right)^{T} B_{u}^{T}\right) E_{i}$

$+\sum_{1 \leq j \leq n_{z}} \tau_{i, j}\left(E_{i}-E_{j}\right)+\tau_{i,\left(n_{z}+1\right)}\left(E_{i}-\Xi^{T} P_{x_{2}} \Xi\right)<0$

$\Xi^{T} P_{x_{2}} \Xi A+A^{T} \Xi^{T} P_{x_{2}} \Xi \sum_{1 \leq j \leq n_{z}} \tau_{n_{z}+1, j}\left(\Xi^{T} P_{x_{2}} \Xi-E_{j}\right)<0$ 
where $1 \leq i \leq n_{z}, \tau_{i, j} \geq 0, \tau_{n_{z}+1, j} \geq 0(1 \leq j \leq$ $\left.n_{z}\right), \tau_{i,\left(n_{z}+1\right)} \geq 0, W \in \mathbb{R}^{n_{y} \times n_{y}}$ is a diagonal matrix, $\gamma$ a positive scalar, $E_{i}=\operatorname{diag}\left(\boldsymbol{e}_{i}\right)$ and $\boldsymbol{e}_{i}$ is the $i$-th base vector of length $n$ and $\Xi=\left[\begin{array}{ll}0 & I_{n-n_{z}}\end{array}\right] \in \mathbb{R}^{\left(n-n_{z}\right) \times n}$.

(iii) Furthermore, it can be shown that the set

$$
B_{U B}=\left\{\boldsymbol{x} \mid V_{z}(\boldsymbol{x}) \leq 1, V_{\boldsymbol{x}}(\boldsymbol{x}) \leq L_{m}^{2}\right\}
$$

is an invariant set and a set of ultimate boundedness which is reached from any initial state and is robust to a bounded disturbance $\boldsymbol{w}$ (4), assuming in addition to (16-18):

$$
\begin{aligned}
& {\left[\begin{array}{l|l}
A_{12}^{T} \tilde{E}_{i} A_{12}-\tau_{m 1} P_{x_{2}} & A_{11}^{T} \tilde{E}_{i} B_{w 1} \\
\hline * & B_{w 1}^{T} E_{i} B_{w 1}-\Gamma_{w 1} I_{n_{w}}
\end{array}\right]<0} \\
& \sum_{1 \leq j \leq n_{w}}\left(\Gamma_{w 1, j j} L_{j}^{2}\right)+\tau_{m 1} L_{m}^{2}-k_{i}^{2}<0, \\
& {\left[\begin{array}{l|l}
\Xi^{T} P_{x_{2}} \Xi A+A^{T} \Xi^{T} P_{x_{2}} \Xi \\
+\tau_{m 2} P_{x_{2}}+\sum_{1 \leq j \leq n_{z}} \tau_{n_{z}+1, j}\left(\Xi^{T} P_{x_{2}} \Xi-E_{j}\right)
\end{array}\right.} \\
& \begin{array}{l}
* \Xi^{T} P_{x_{2}} \Xi B_{w} \\
\sum_{1 \leq j j \leq n_{w}}\left(\Gamma_{w 2, j j} L_{j}^{2}\right)-\tau_{m 2} L_{m}^{2}<0
\end{array}
\end{aligned}
$$

for $1 \leq i \leq n_{z} ; \tau_{m 1}, \tau_{m 2} \geq 0$ and diagonal matrices $\Gamma_{w 1}, \Gamma_{w 2} \geq 0$ where $\tilde{E}_{i}=\operatorname{diag}\left(\tilde{\boldsymbol{e}}_{i}\right)$ and $\tilde{\boldsymbol{e}}_{i}$ is the $i$-th base vector of length $n_{z}$. Assuming for the initial state $\boldsymbol{x}(0) \in B_{U B}$ the output constraint for $\boldsymbol{z}$ is ensured. $\diamond$

Please find the proof in the appendix of this paper.

Remark 1. Assuming the variables of the matrix inequality of (16) are $M_{1}, M_{2}, P_{s}, W$ and $\gamma$ then it is easily seen that (16) is not linear or bilinear in these variables but third order. Furthermore, the variables $M_{1}$ and $M_{2}$ are also variables of the $n_{z}-1$ matrix inequalities of (17). Due to this coupling of (16) and (17), it is not possible to use a change of variable approach employing linear transformation techniques to decrease the order of the three coupled matrix inequalities of (16)-(18). However, the Schur complement can be used to obtain from (16) an equivalent bilinear expression:

$\left[\begin{array}{l|l|l|l|}P_{s} A+P_{s} B_{u} M_{1} C_{y}+A^{T} P_{s} & P_{s} B_{u} & \begin{array}{l}C_{y}^{T} W \\ +P_{s} B_{u} M_{2}\end{array} & C_{y}^{T} M_{1}^{T} \\ +C_{y}^{T} M_{1}^{T} B_{u}^{T} P_{s} & -\gamma I & 0 & 0 \\ \hline * & * & -2 W & M_{2}^{T} \\ \hline * & * & * & -\gamma I\end{array}\right]<0$

Thus, it is now possible to use bilinear matrix inequality solution methods (Fukuda and Kojima, 2001; Goh et al., 1995; Balakrishnan and Boyd, 1992) to solve the matrix inequalities of (17)-(18) and (22) to minimize the $\mathcal{L}_{2}$-gain $\gamma$.

Remark 2. Note that for some nominal control systems, it may not be necessary to introduce the override term $M_{1} \boldsymbol{y}+\boldsymbol{u}_{o 1}$ as $\gamma \rightarrow 0$. Hence, in this case, only the high gain component $\boldsymbol{u}_{o 2}(5)$ acts to retain the amplitude constraint for $z$. The non-linear override controller term $\boldsymbol{u}_{o 2}$ acts due to the discontinuous character so that the amplitude limits for $z$ are always retained (for suitable initial value of $\boldsymbol{x}(0)$ and bounded $\boldsymbol{w})$, although $\boldsymbol{u}_{o 2}$ is only used for $\left|z_{i}\right|>1, i=1, \ldots, n_{z}$. However, this precludes 'ideal' conditions: the non-linear term $\boldsymbol{u}_{o 2}$ has to be able to switch infinitely fast. In a practical discrete implementation this is usually not possible so that the output constraint will always be violated.
However, the degree of violation is bounded by a given control system dependent bound, which can be made arbitrarily small.

\section{A SIMULATION EXAMPLE}

For validation of the override control technique, the model $G=\left(A_{G}, B_{G}, C_{G}\right)$ of a well known furnace example (Edwards and Spurgeon, 1998, Chapter 9) is used:

$$
\begin{aligned}
& A_{G}=\left[\begin{array}{rrrr}
-0.0186 & -0.0065 & 0.0190 & 0.0129 \\
0.0026 & -0.1354 & 0.0310 & 0.0040 \\
-0.0972 & 0.0695 & -0.1273 & 0.0530 \\
-0.0193 & -0.0155 & -0.1121 & -0.4934
\end{array}\right], \\
& B_{G}=\left[\begin{array}{rr}
0 & 0 \\
0 & 0 \\
0 & -0.0960 \\
0.4969 & 0.0453
\end{array}\right] \text {, } \\
& C_{G}=\left[\begin{array}{rrrr}
0.6707 & -0.1085 & -0.0286 & 0.0086 \\
-0.2750 & -0.1933 & -0.2175 & 0.0060
\end{array}\right]
\end{aligned}
$$

This multi-variable model represents a practically existing furnace containing at one of the side walls a burner for which the fuel supply (first actuator input) and the oxygen trim-signal (first output measurement), a signal proportional to the fuel/air ratio, can be adjusted. Hence, one important task is to control the temperature of this furnace. At the same time, ecological constraints necessitate to limit the oxygen concentration (second output measurement) in the gaseous combustion products which are released through the flue in the roof. The assumption here is that this model is valid for the operation point defined by the output pair $\left[675^{\circ} \mathrm{C}, 3 \%\right]$. Although the furnace has been practically tested for an oxygen concentration range of [2\%, 11\%] (Edwards and Spurgeon, 1998, Chapter 9), it is here the task to to retain the oxygen concentration in the interval $[(3-2) \%,(3+2) \%]$ to take into consideration the need for lower oxygen concentration. For instance, a concentration of about $3 \%$ is considered by Casaca and Costa (2003). Note that the interval $[(3-2) \%,(3+2) \%]$ is symmetric with respect to the oxygen concentration operational point. For control, the temperature follows a tracking demand in the interval $\left[675^{\circ} \mathrm{C}, 800^{\circ} \mathrm{C}\right]$. The oxygen concentration is a measure for the efficiency of the furnace, which is directly related to the air supply of the furnace: An excess of supplied air causes an undue loss of heat through the flue, while a lack of air will lead to an incomplete combustion.

A linear $H_{\infty}$-controller $K \equiv\left(A_{K}, B_{K}, C_{K}, C_{K}\right)$ has been designed, weighting the sensitivity $S$ and the controller output $K S$, ensuring rise/settling times of about $10 \mathrm{sec} .$. The closed loop system including the $H_{\infty}$-controller reduced to 4 th order is represented by:

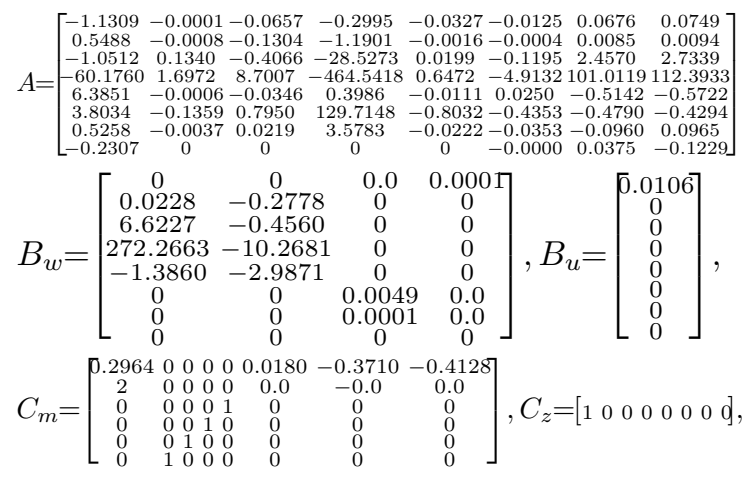


Thus, only the oxygen trim signal is used to retain the constrained oxygen concentration in the required interval of $[1 \%, 5 \%]$. For the override controller design a local optimization approach was found sufficient, minimizing the $\mathcal{L}_{2}$-gain $\gamma$ to $\gamma<277$ (15):

$$
\begin{aligned}
& M_{1}=\left[\begin{array}{llll}
-3.5058 & 0 & 0.915328 .01112 .3139 & 1.3166
\end{array}\right], \\
& M_{2}=\left[\begin{array}{lll}
-0.9043-83.7869 \cdot 10^{10} & 0.3898 & 10.261-0.47161 .4245
\end{array}\right] \cdot 10^{-10}
\end{aligned}
$$

while for the considered temperature demand range a value of $k_{1}=50$ was found suitable. The value $\mu=0.99$ ensures that the non-linear override term $u_{o 1}(5)$ is used only for a very short moment. When comparing in Figure 2 the complementary sensitivity response for the nominal closed loop $(u=0)$ (1) and the controlled system using the linear override component only ( $\left.u=M_{1} \boldsymbol{y}\right)(5)$, it is observed that the effect of $M_{1} \boldsymbol{y}(5)$ is minor.

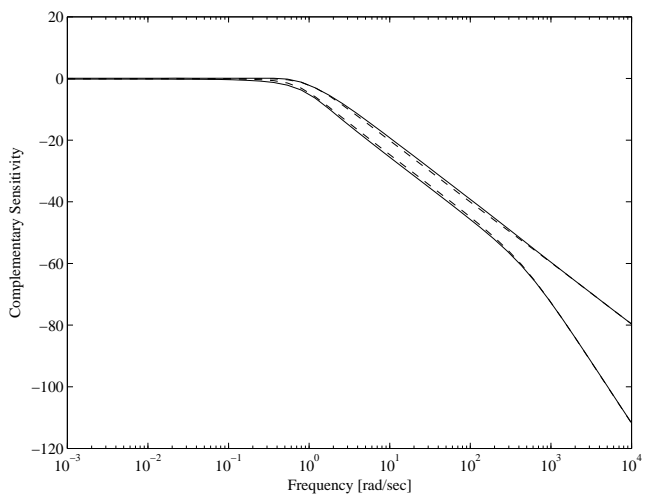

Fig. 2. Nominal ( $u=0$, dashed) and modified ( $u=M_{1} \boldsymbol{y}$, line) complementary sensitivity response

The complete override controller, $\left(u=M_{1} \boldsymbol{y}+\boldsymbol{u}_{o 1}+\right.$ $\boldsymbol{u}_{o 2}$ ), of (5) was tested using a fast sampling approach, i.e. the overall control system was simulated as a discrete algorithm using a high sampling frequency of $200 \mathrm{~Hz}$. The control system (Figure 3) in response to a series of slow temperature demand changes demonstrates that the override scheme of (5) is indeed effective for enforcement of a strict output limitation of the oxygen concentration (see Figure 4). However,

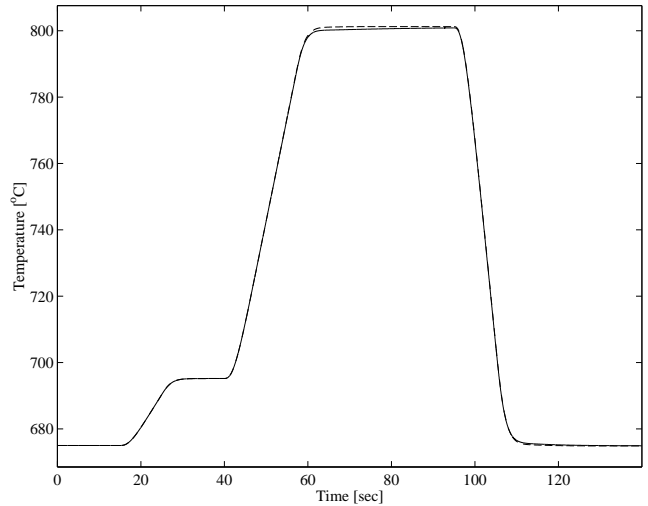

Fig. 3. Temperature in response to step demands employing override controller (5) (line) and linear override component only (dashed)

when comparing the override scheme $\left(u=M_{1} \boldsymbol{y}+\right.$ $\left.\boldsymbol{u}_{o 1}+\boldsymbol{u}_{o 2}\right)$ of (5) with the mildly modified controller $\left(u=M_{1} \boldsymbol{y}\right)$, it is seen that once the oxygen concentration hits the prescribed limit, the step response of the furnace temperature (Figure 3 ) is slightly slowed down for the override scheme. Note also, that the override controller shows an increased control effort (see the trim signal (Figure 5)) once the constrained output is

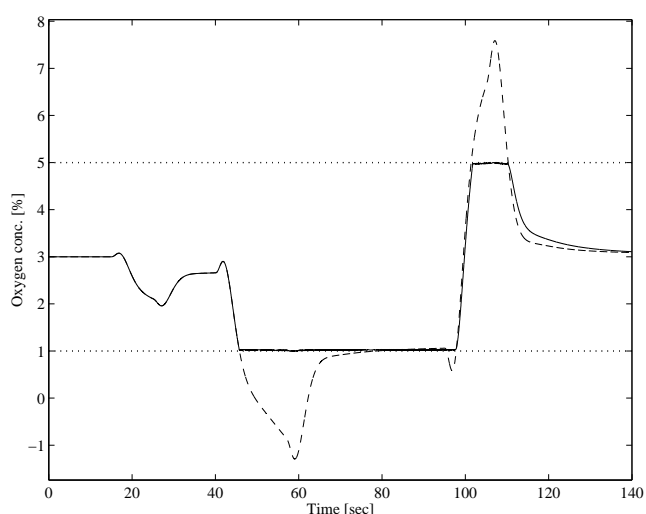

Fig. 4. Oxygen concentration in response to temperature step demands employing override controller (5) (line) and linear override component only ( $u=M_{1} \boldsymbol{y}$, dashed)

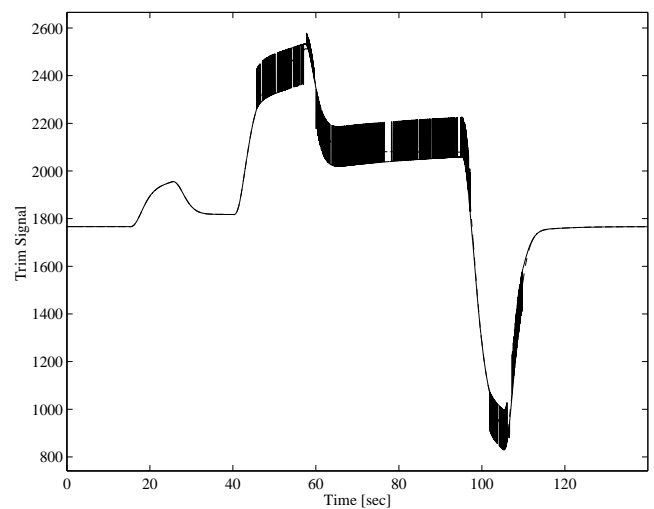

Fig. 5. Oxygen trim signal for temperature step demands employing override controller (5) (line) and linear override component only ( $u=M_{1} \boldsymbol{y}$, dashed)

at the allowed limit. In particular a high frequency component can be observed.

\section{CONCLUSIONS}

The paper has presented a nonlinear override control scheme which allows to limit the output measurement to a given interval which is symmetric to the operation point. The override controller consisting of a linear, a switched linear and a non-linear high-gain component is designed so that the nominal loop is affected as little as possible. For this, the map of the exogenous outputs to the linear and the switched linear override controller term is $\mathcal{L}_{2}$-gain minimized using a set of bilinear matrix inequality conditions.

The non-smooth max-Lyapunov function is used to prove asymptotic stability for closed loop control without the effect of exogenous signals while all output constraints are satisfied for a given invariant set defined by the controller designer.

A simulation example for temperature control of a linear model for a practically existing furnace has shown that the override controller is effective for the considered demand profile.

\section{REFERENCES}

Balakrishnan, V. and S. Boyd (1992). Global optimization in control system analysis and design. In: Control and Dynamic Systems: Advances in Theory and Applications (C.T. Leondes, Ed.). Vol. 53. Academic Press, New York.

Bemporad, A., F. Borrelli and M. Morari (2003). Min-max control of constrained uncertain discrete-time linear systems. IEEE Trans. Aut. Contr. 48(9), 1600-1606.

Blanchini, F. (1995). Nonquadratic Lyapunov functions for robust control. Automatica 31(3), 451-461. 
Boyd, S., L. El Ghaoui, E. Feron and V. Balakrishnan (1994). Linear Matrix Inequalities in System and Control Theory. SIAM, Philadelphia.

Casac, C. and M. Costa (2003). Co-combustion of biomass in a natural gas fired furnace. Combust. Sci. \& Technol. 175, 19531977.

Clarke, F. H., Yu. S. Ledyaev, R. J. Stern and P. R. Wolenski (1998). Nonsmooth Analysis and Control Theory. Springer, New York.

Craven, B. D. (1982). Lebesgue measure and Integral. Pitman Publ. Inc.

Edwards, C. and S. K. Spurgeon (1998). Sliding Mode Control: Theory and Application. Taylor \& Francis, London.

Filipov, A. F. (1964). Differential equations with discontinuous right-hand side. Amer. Math. Soc. - Translations 42, 199-231.

Fukuda, M. and M. Kojima (2001). Branch-and-cut algorithms for the bilinear matrix inequality eigenvalue problem. Comput. Optim. \& Appl. 19, 79-105.

Gilbert, E. G. and K. T. Tan (1991). Linear systems with state and control constraints: the theory and application of maximal output admissible sets. IEEE Trans. Aut. Contr. 36(9), 1008 $-1020$.

Glattfelder, A.H. and W. Schaufelberger (1988). Stability of discrete override and cascade-limiter single-loop control systems. IEEE Trans. Aut. Contr. 33(6), 532-540.

Glattfelder, A.H. and W. Schaufelberger (2003). Control Systems with Input and Output Constraints. Springer, Berlin.

Goh, K.-C., M. G. Safonov and G. P. Papassiloupoulos (1995). Global optimization for the biaffine matrix inequality problem. J. Glob. Optim. 7, 365-380.

Herrmann, G. (2000). Discretization of non-linear controls with application to robust, sliding-mode-based control systems. PhD-th. Univ. Leicester.

Herrmann, G., S. K. Spurgeon and C. Edwards (1999). Discretization of sliding mode based control schemes. In: Proc. 38th CDC, Phoenix. pp. 4257-4262.

Mareczek, J., M. Buss and G. Schmidt (2000). Sufficient conditions for invariance control of a class of nonlinear systems. In: Proc. 39th CDC. pp. $1900-1905$.

Mareczek, J., M. Buss and M. W. Spong (2001). Invariance control of normal forms with input driven internal dynamics. In: Proc. ACC. pp. 3648 - 3653 .

Mareczek, J., M. Buss and M. W. Spong (2002). Invariance control for a class of cascade nonlinear systems. IEEE Trans. Aut. Contr. 47(4), 636-640.

Saberi, A., J. Han and A. A. Stoorvogel (2002). Constrained stabilization problems for linear plants. Automatica 38, 639-654.

Turner, M. C. and I. Postlethwaite (2002). Output violation compensation for systems with output constraints. IEEE Trans. Aut. Contr. 47(9), 1540-1546.

Wolff, J. and M. Buss (2004). Invariance control design for nonlinear control affine systems under hard state constraints. In: Proc. 6th (IFAC) NOLCOS, Stuttgart. pp. 711-716.

\section{APPENDIX - PROOF OF THEOREM 1}

It is easily verified that the extended Lyapunov constraint of (15) is satisfied for the matrix inequality condition of (16). Similarly, a matrix inequality condition is formulated for the stability constraint of (9) and (10) for $\mu<V_{x}$ assuming $\boldsymbol{w}=0$. For this, the $\mathcal{S}$-procedure (Boyd et al., 1994) is used to include the following relations for $V_{i}=V_{\boldsymbol{x}},\left(i<n_{z}+1\right)$ into the stability constraint $\frac{d V_{i}}{d t}<0$ of (10):

$$
\boldsymbol{x}^{T} E_{i} \boldsymbol{x}-\boldsymbol{x}^{T} E_{j} \boldsymbol{x} \geq 0, i \neq j
$$

and

$$
\boldsymbol{x}^{T} E_{\boldsymbol{z}} \boldsymbol{x}-\boldsymbol{x}^{T} \Xi^{T} P \Xi \boldsymbol{x} \geq 0,
$$

These relations follow from $V_{i}=V_{x}$. Thus, for $1 \leq$ $i \leq n_{z}$ the $\mathcal{S}$-procedure implies the matrix inequality of (17). For $V_{n_{z}+1}=V_{x}$, the following relation is to be considered:

$$
\boldsymbol{x}^{T} \Xi^{T} P \Xi \boldsymbol{x}-\boldsymbol{x}^{T} E_{i} \boldsymbol{x} \geq 0
$$

implying the matrix inequality constraint of (18). Note that the second non-linear override component $\boldsymbol{u}_{o 2}$ improves the stabilizing condition of (17) and acts for $V_{z} \geq 1$ for which reason it has not been considered in this case.
Ultimate boundedness can be implied from analyzing $\frac{d V_{x}}{d t}$ for bounded exogenous signal $\boldsymbol{w}$. Considering $V_{x}=V_{n_{z}+1}$ it follows using the same rationale as before:

$$
\begin{aligned}
& \frac{d V_{n_{z}+1}}{d t} \leq \boldsymbol{x}^{T}\left(\Xi^{T} P_{x_{2}} \Xi A+A^{T} \Xi^{T} P_{x_{2}} \Xi+\right. \\
& \left.\sum_{1 \leq j \leq n_{z}} \tau_{n_{z}+1, j}\left(\Xi^{T} P_{x_{2}} \Xi-E_{j}\right)\right) \boldsymbol{x}+2 \boldsymbol{x}^{T} \Xi^{T} P_{x_{2}} \Xi B_{w} \boldsymbol{w}
\end{aligned}
$$

As the set $B_{U B}$ is supposed to be a set of ultimate boundedness, in particular the set $\left\{\boldsymbol{x} \mid V_{\boldsymbol{x}}(x) \leq\right.$ $\left.L_{m}^{2}\right\}$, it is necessary that $\frac{d V_{x}}{d t}$ remains decreasing for $V_{n_{z}+1}(\boldsymbol{x})>L_{m}^{2}$ and $V_{\boldsymbol{x}}=V_{n_{z}+1}$. Hence, the following constraint

$$
\boldsymbol{x}_{2}^{T} P_{\boldsymbol{x}_{2}} \boldsymbol{x}_{2}-L_{m}^{2}>0
$$

is considered for the inequality (22) using the $\mathcal{S}$ procedure while the boundedness constraint of (4) can be included using:

$$
\sum_{1 \leq i \leq n_{w}}\left(\Gamma_{w 2, i i} L_{i}^{2}\right)-\boldsymbol{w}^{T} \Gamma_{w 2} \boldsymbol{w} \geq 0
$$

Combining these relationships (23) and (24) with (22), the linear matrix inequality conditions of (21) are obtained. Furthermore, for $V_{\boldsymbol{x}}=V_{i}\left(0 \leq i \leq n_{z}\right)$ follows from (17):

$$
\frac{d V_{i}}{d t} \leq\left|\boldsymbol{x}_{1} \tilde{E}_{i}\right|\left(\left|\tilde{E}_{i} B_{w 1} \boldsymbol{w}\right|-k_{i}\right)
$$

Hence, if $\left(\left|\tilde{E}_{i} B_{w 1} \boldsymbol{w}\right|-k_{i}\right)<0$ then ultimate boundedness with respect to the set $\left\{\boldsymbol{x} \mid V_{\boldsymbol{x}}(\boldsymbol{x}) \leq L_{m}^{2}\right\}$ follows as $\frac{d V_{x}}{d t}<0$ almost everywhere in $t$ is ensured outside the set $\left\{\boldsymbol{x} \mid V_{\boldsymbol{x}}(\boldsymbol{x}) \leq L_{m}^{2}\right\}$ and for bounded $\boldsymbol{w}$. It will be shown that the linear matrix inequalities of (19) and (20) are sufficient to satisfy this.

Amplitude boundedness of $z$ follows from the analysis of (12) for $V_{z}>1$ considering a bounded disturbance $\boldsymbol{w}$. For $V_{i}=V_{z},\left(1 \leq i \leq n_{z}\right)$, it follows as for (17) using the $\mathcal{S}$-procedure that if the following inequality holds:

$$
\begin{aligned}
& \boldsymbol{x}_{1}\left(\tilde{E}_{i}\left(A_{11}+B_{u 1}\left(M_{1}+M_{2}\right) C_{y}\right)\right. \\
& \left.\quad+\left(A_{11}^{T}+C_{y}^{T}\left(M_{1}+M_{2}\right)^{T} B_{u 1}^{T}\right) \tilde{E}_{i}+\sum_{1 \leq j \leq n_{z}} \tau_{i, j}\left(\tilde{E}_{i}-\tilde{E}_{j}\right)\right) \boldsymbol{x}_{1} \\
& \quad+\left|\boldsymbol{x}_{1} \tilde{E}_{i}\right|\left(\left|\tilde{E}_{i} B_{w 1} \boldsymbol{w}+\tilde{E}_{i} A_{11} \boldsymbol{x}_{2}\right|-k_{i}\right)<0
\end{aligned}
$$

then $\frac{d V_{i}}{d t}<0$ for $V_{i}=V_{z}$. The first quadratic component is negative for $V_{z}>1$ as (17) is shown to be negative definite. Thus, it remains to choose $k_{i}$ large enough so that the term (see (19))

$$
\left(\left|\tilde{E}_{i} B_{w 1} \boldsymbol{w}+\tilde{E}_{i} A_{12} \boldsymbol{x}_{2}\right|^{2}-k_{i}^{2}\right)<0
$$

is negative for bounded $\boldsymbol{x}_{2}$ (13) and bounded disturbance $w(4)$. Using again the $\mathcal{S}$-procedure to extend (26), it follows that if (19) and (20) are satisfied, then $\left(\left|\tilde{E}_{i} B_{w 1} \boldsymbol{w}+\tilde{E}_{i} A_{12} \boldsymbol{x}_{2}\right|-k_{i}\right)<0$ and $V_{z}$ is ultimately smaller than 1 for small enough $V_{n_{z}+1}\left(\boldsymbol{x}_{2}\right)$ (or $\left.\left\|\boldsymbol{x}_{2}\right\|\right)$ and bounded $\boldsymbol{w}$. This also implies that $B_{U B}$ and $\left\{\boldsymbol{x} \mid V_{\boldsymbol{x}}(\boldsymbol{x}) \leq L_{m}^{2}\right\}$ are sets of ultimate boundedness as well as invariant sets for the override control scheme. Thus, the strict limits on the output $z$ are ensured assuming for the initial states $\boldsymbol{x}(0) \in B_{U B}$. 